\title{
Bayesian Preference Inference in Dialogue
}

\author{
Asya Achimova \\ asya.achimova@uni-tuebingen.de \\ Ella I. Eisemann \\ ella-isabel.eisemann@student.uni-tuebingen.de \\ Martin V. Butz \\ martin.butz@uni-tuebingen.de \\ Neuro-Cognitive Modeling Group \\ Department of Computer Science and Department of Psychology \\ Faculty of Science, University of Tübingen \\ Sand 14, 72076 Tübingen, Germany \\ https://cm.inf.uni-tuebingen.de
}

\begin{abstract}
An utterance is referentially ambiguous if it has several potential referents. Observing how listeners make choices among those referents can reveal their hidden beliefs and preferences, besides giving hints at their responding processes. We asked subjects to observe how one of the objects is chosen following a possibly ambiguous utterance and to infer which preferences the listener may have had in mind when choosing the particular object. In order to adjust this interaction to a dialogue-like setting, we extended the traditional one-shot reference game to a round of 4-trial games. Moreover, we modeled the process within the Rational Speech Act framework, implementing iterative inference over multiple trials, where posteriors from previous trials carry over to the next trial as priors. The model predicts human inference behavior better than a baseline model as well as better than a non-iterative model. The results imply that, in principle, humans are able to compute Bayesian-like inferences in dialogue, learning about the beliefs and preferences of others in an iterative, cumulative manner.

Keywords: ambiguity; iterative learning; pragmatics; information gain; eventpredictive cognition; Rational Speech Act models; social intelligence
\end{abstract}

\section{Introduction}

Social interactions rely on speakers being able to simulate the listener's thought processes, including - to some extent - the listener reasoning about why the speaker chose to say the things she did. For communicative behavior to be adaptive, interlocutors need to continuously update predictive model components of each other. These models include, among other things, understanding the state of each other's knowledge, beliefs, and preferences. During conversations, we actively seek to update the information state not only about the world but also about each other. Such interpretation of communicative behavior fits within a broader perspective on active inference as a core principle on 
which the human mind operates (Friston \& Frith, 2015; Hohwy, 2013; Clark, 2016). In this paper, we adopt the predictive mind perspective as it applies to social interactions.

We argue that ambiguity, which is omnipresent in communication, provides a learning platform where conversation partners can gain better understanding of each other. This work presents a considerable departure from a classic view on ambiguity, which treats it as an inconvenient side effect of a language system (Grice, 1975; Chomsky, 2002). Within that paradigm, ambiguous utterances prevent an accurate transfer of information between interlocutors and therefore obscure communication.

Despite the apparent negative consequences associated with the use of ambiguous utterances, agents rarely avoid ambiguity even in situations where it would be communicatively appropriate (Wasow, 2015; Ferreira, 2008). Instead, speakers rely on listeners to fill in the missing information by identifying the interpretation that is most coherent within the current discourse. Piantadosi, Tily, and Gibson (2012) show via statistical modeling that ambiguity is an essential product of an efficient communication system: it allows to reuse lightweight pieces of language instead of giving a fully specified description of a situation. Ambiguous descriptions save the effort on the speaker's side, while relying on the listener's ability to interpret the ambiguous utterances within context.

We propose that ambiguity of reference creates a space of alternatives, such that the choice of one of the available alternatives becomes a meaningful event in itself. This idea is consistent with the attribution theory, which captures the human ability to interpret each other's behavior as driven by motives, intentions, and goals (Jones \& Davis, 1965; Kelley, 1967; Kelley \& Stahelski, 1970). In a naturalistic conversation, where speakers take turns, ambiguous utterances open interpretation spaces and the resulting interpretation choices dynamically and mutually reveal individual opinions, beliefs, and preferences.

In order to draw inferences about individual predispositions, the speaker needs to reason pragmatically about the listener. We use the paradigm of references games, as developed in Frank, Goodman, Lai, and Tenenbaum (2009), to model this choice process, and test whether human subjects are able to infer what properties of objects determined a particular course of events. In the course of a classic reference game, a speaker wants to signal a particular object to the listener. In order to do so, the speaker is allowed to use one-word utterances to refer to one of the objects (e.g. Frank \& Goodman, 2012). The task of the listener is to infer which of the available objects is the most likely referent. The listener reasons about the process that generated the utterance, assuming that the speaker uses the utterance that is the most efficient to signal a particular object.

For example, consider a scenario in Figure 1. Suppose a speaker produces a singleword utterance "blue" - meaning: choose a blue object - creating referential ambiguity for the listener, that is, offering a choice between a blue square and a blue circle. Suppose further that, upon hearing "blue", the listener selects the blue circle. In observing this choice, the speaker learns something about the private thoughts of the listener: what made her select the blue circle instead of the blue square? If the listener's choice was driven by purely pragmatic considerations modeled in Frank and Goodman (2012), the listener should have selected the blue square, since if the speaker had intended to refer to the circle, she could have used the more efficient, non-ambiguous utterance "circle". Since she did not say "circle", however, she must have referred to the square. Yet, in our situation, the listener did pick the circle following the utterance "blue". Perhaps there is another choice strategy that the listener is using: the circle might be more salient to the listener, the listener has a preference for circles, or the listener may 

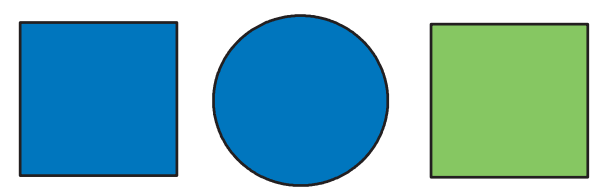

Figure 1: A simple reference game scenario from Frank and Goodman (2012). In the game, speakers are confronted with a collection of objects, which determine the current scenario $S$, where $S=\{$ solid blue square, solid blue circle, solid green square $\}$ in the depicted example. A speaker may choose a single-word utterance $u$ to signal one of the objects $s \in S$ to a listener. In the shown scenario, the following set of utterances is available: $U=\{$ "solid", "blue", "green", "square", "circle" $\}$.

believe that the speaker has a preference for circles; there may even be mutual agreement that circles are to be preferred when possible. Importantly, by observing how the listener resolves the ambiguity in reference, the speaker can learn something about the private thoughts of the listener.

In a modified version of reference games, we created situations where a speaker picks an utterance and watches a simulated listener choose one of the objects. The task of the participant is to infer what preferences of the listener made her pick that particular object. In order to avoid an impression that the choice of an object might be random, we allow 4 consecutive trials that focus on the preferences of one particular person. Participants then track the object choices and update their understanding of the listener's preferences across a series of trials. We hypothesized that subjects use a Bayesian inference process: they take into account their existing priors of the listener's preferences as they accumulate more evidence.

\section{Modeling}

The recursive interactions between the speaker and the listener are modeled within the Rational Speech Act (RSA) framework (Frank \& Goodman, 2012; Goodman \& Stuhlmüller, 2013; Franke \& Jäger, 2016; Goodman \& Frank, 2016, cf. a gametheoretic approach of recursive reasoning in Franke, 2009). The model formalizes a state space, or scenario, $S$ in the form of a particular set of objects (e.g. Figure 1) and the corresponding utterance space $U$. The utterance space contains all possible utterances, which correspond to object features that are present in a particular scenario $S$.

At the base of the reasoning process, there is a hypothetical, naïve literal listener $L_{0}$, who hears an utterance $u \in U$ and attempts to infer the object $s \in S$ that $u$ is meant to reference. $L_{0}$ performs this inference by conditioning on the literal semantics of $u$, $\llbracket u \rrbracket(s)$, which returns true (i.e., 1) for those objects that contain the uttered feature and false (i.e., 0), otherwise. As a result, object choice probabilities for the literal listener can be computed by:

$$
P_{L_{0}}(s \mid u) \propto[[u]](s),
$$

essentially returning a uniform distribution over those objects in $S$ that contain the uttered feature $u{ }^{1}$

\footnotetext{
${ }^{1}$ Note that the context $S$ is typically not made explicit, but rather treated implicitly in the specification of
} the model. 
One layer up, the speaker $S_{1}$ observes the state $S$ and is assumed to have the intention to refer to a particular object $s \in S$. $S_{1}$ chooses an utterance $u$ on the basis of its expected utility for signaling $s$ in the scenario $S$, which is determined by the $\log$-likelihood of this particular object choice $U_{S_{1}}(u ; s):^{2}$

$$
U_{S_{1}}(u ; s)=\log \left(P_{L_{0}}(s \mid u)\right) .
$$

Depending on a "greediness" factor $\alpha$, the speaker chooses a particular utterance $u$ with a probability that is exponentially proportional to the utility estimate:

$$
P_{S_{1}}(u \mid s) \propto \exp \left(\alpha \cdot U_{S_{1}}(u ; s)\right) .
$$

At the top layer of the vanilla RSA model, the pragmatic listener $L_{1}$ infers posteriors over $s$ on the basis of some observed utterance $u$. However, unlike $L_{0}, L_{1}$ updates beliefs about the world by reasoning about the process that generated $u$, namely the utterance choice of speaker $S_{1}$. In other words, $L_{1}$ reasons about which object $s$ would have been most likely led $S_{1}$ to utter $u$ given the scenario $S$ :

$$
P_{L_{1}}(s \mid u) \propto P_{S_{1}}(u \mid s) \cdot P(s) .
$$

Frank and Goodman (2012) tested the predictions of RSA against behavioral data from reference games, as in Figure 1. They found a strong correlation between model predictions and behavioral data, confirming the validity of their model of pragmatic reasoning in reference games (see also Qing \& Franke, 2015 for a fuller exploration of the modeling choices).

\subsection{Pragmatic social inference RSA model}

Our model builds on the vanilla version of RSA, modifying the listener's state prior $P(s)$ and enhancing the reasoning process towards a social component, yielding a pragmatic social inference RSA model (PSIRSA). By changing $P(s)$ to a non-uniform distribution, we essentially model prior beliefs of which object the speaker is more likely to refer to, or-when viewed from a more self-centered perspective-which prior object feature preferences $f$ the listener may have. For example, the listener may like blue things, such that she may be more likely to choose the blue square instead of the green one when hearing the utterance "square" in the scenario shown in Figure 1. As a result, when a pragmatic speaker produces utterance $u$ and observes the listener's referent choice $s$, the speaker may infer posteriors over possible feature preferences, attempting to explain the observed object choice in this way.

We introduce two more modifications to the original version of RSA. First, our model relies on fewer layers of reasoning. Recently, it has been shown that even in the original, simpler reference games, fewer layers of reasoning often perform equally well or better than more complex RSA-based models (Sikos, Venhuizen, Drenhaus, \& Crocker, 2019). Accordingly, PSIRSA removes the reasoning about alternative utterances and allows the pragmatic speaker to directly tap into the (expected) interpretation of $L_{0}$, augmenting the literal listener's choice likelihoods with the feature-preferencedependent object prior $P(s \mid f)$ :

$$
P_{L_{0}}(s \mid u, f) \propto[[u]](s) \cdot P(s \mid f) .
$$

\footnotetext{
${ }^{2}$ The original model in Frank and Goodman (2012) also includes a term for the utterance cost, $C(u)$. We ignore the term here since we assume uniform cost over all utterances.
} 
The pragmatic speaker $S_{1}$ then reasons directly about the modified literal listener $L_{0}$ :

$$
P_{S_{1}}(f \mid u, s) \propto P_{L_{0}}(s \mid u, f) \cdot P(f) .
$$

As a result, PSIRSA ignores any indirect pragmatic reasoning considerations about which object the speaker may refer to given an utterance and a particular object constellation. It simply assumes that all objects may be chosen that match the utterance, modifying these choice options dependent on the feature-preference-dependent object choice priors. The corresponding utterance-selection model simplifies the reasoning process accordingly:

$$
P_{S_{1}}(u) \propto \sum_{s: \llbracket u \rrbracket(s)=1} P_{L_{0}}(s \mid u, f) \exp \left(\lambda \cdot \operatorname{KL}\left(P(f) \| P_{S_{1}}(f \mid u, s)\right)\right) .
$$

The utterance choice process is aimed at maximizing the distance between the flat prior distribution of listener preferences and the posterior that can be obtained having observed a particular object choice. We formalize the distance between these two distributions as Kullback-Leibler divergence (KL).

Second, we model reasoning as an iterative process where a learner accumulates and updates her understanding of another person's beliefs over a set of trials. A learner starts with a flat prior over possible feature preferences and infers posteriors upon hearing an utterance and observing an object choice. Then the model passes the obtained posterior distribution of preferences as the preference prior to the next trial, implementing an iterative Bayesian inference process.

\subsection{Free parameter optimization}

We fit the model parameters at the group and individual levels by optimizing the KL divergence between the data and the model predictions:

$$
\operatorname{KL}\left(P_{\text {data }}(f \mid u, s) \|\left(P_{\text {model }}(f \mid u, s)\right),\right.
$$

where $P_{\text {data }}(f \mid u, s)$ specifies a participant's normalized response value, which offers empirical estimates of the feature-preference posterior given object scene $S$, a particular utterance choice $u$, and the consequent object choice $s$. Note that these conditional probability values are directly set by means of the adjustable sliders by each participant in each trial (cf. Figure 2 and details below). As a result, the participants directly estimate a probability mass (i.e. a discrete density) over feature preferences in each trial. Since no conclusions can be drawn concerning feature values that are not present in the scene, we ignored the respective feature preference estimates, and renormalize the slider values. The resulting probability mass is then compared with $P_{\text {model }}(f)$ $u, s)$, which specifies the corresponding model posterior $P_{S_{1}}(f \mid u, s)$, using the KL divergence function.

By minimizing the summed KL divergence between the empirical and modelpredicted preference posteriors over all considered trials, we essentially maximize the model fit to the participants' data. Moreover, we can use the minimized KL divergence values to calculate the $G^{2}$-statistic and perform the likelihood ratio test for nested models, since $G^{2}$ values are approximately chi-square distributed (Lewandowsky \& Farrell, 2011). Individual vs. global parameter fitting allows us to explore potential differences between participants. In the case of individual model parameter optimization, parameters were optimized for each individual participant separately, determining the summed 
KL divergence with respect to the participant-specific set of trials. In the case of global model parameter optimization, all trials of all participants were used to determine the summed KL divergence.

The softness parameter $\gamma$ regulates the strength of individual feature preferences $f$ :

$$
P(s \mid f) \propto\left\{\begin{array}{ll}
1+\gamma, & \text { if } s \text { contains } f \\
\gamma, & \text { otherwise }
\end{array},\right.
$$

controlling the choice probability of those objects $s$ that contain feature $f$ compared to those that do not. A value of $\gamma=0$ models a hard preference choice; in this case, the speaker always chooses one of the preferred objects. On the other hand, when $\gamma \rightarrow \infty$, the choice prior becomes uniform over all objects, thus ignoring feature preferences. For example, consider a scenario in Figure 2, where the listener picked a striped object following the utterance "circle". If her $\gamma=0$, she should adjust the "striped" slider to 1 and set other sliders to 0 if we assume she has not previously learned anything about the relative ranking of the other values. If her $\gamma=1$, the model predicts that she sets the "striped" slider to 0.5 and the other slider values to 0.25 . In that case, we can say that her preferences became softer, i.e. less categorical or strict. We use $\gamma=0-$ that is, hard preferences-as the default model value.

Finally, we allow for the possibility of noise in our human data introduced by participants not following instructions. Parameter $\beta$ models the possibility that listeners choose objects that do not pass the semantic filter of the literal listener, allowing for non-literal interpretations that result in choosing objects whose features do not match the received utterance $u$. The computation is equivalent to the softness parameter above, in this case softening the object choices of the literal listener $L_{0}$ towards a uniform choice over all objects present.

Again, $\beta=0$ models a hard object choice-that is, full obedience to the uttered instruction $u$-while $\beta \rightarrow \infty$ models a uniform object choice-that is, full ignorance of $u$.

\section{Experiment}

The experiment was designed to address three questions: first, whether speakers are able to pick ambiguous utterances that could create a potentially informative learning situation. In such a situation, the listener's object choice would reveal her preferences. Second, we asked whether participants are able to infer the preferences of the listener. Finally, we were interested in whether participants are able to use the information about the listener's preferences that they accumulated over previous trials. This passing of information over trials helps the learners base their guesses on an informative rather than an uninformative flat prior. If their informative prior is accurate, the posterior estimate is also expected to improve, resulting in overall learning success. In the results below we focus on the second and third question. However, the data also contains strong indications that many, but clearly not all, participants were able to strategically pick ambiguous utterances over less ambiguous ones.

\subsection{Participants}

We recruited 100 participants with US IP addresses through Amazon.com's Mechanical Turk crowd sourcing service. Subjects were compensated for their participation. 


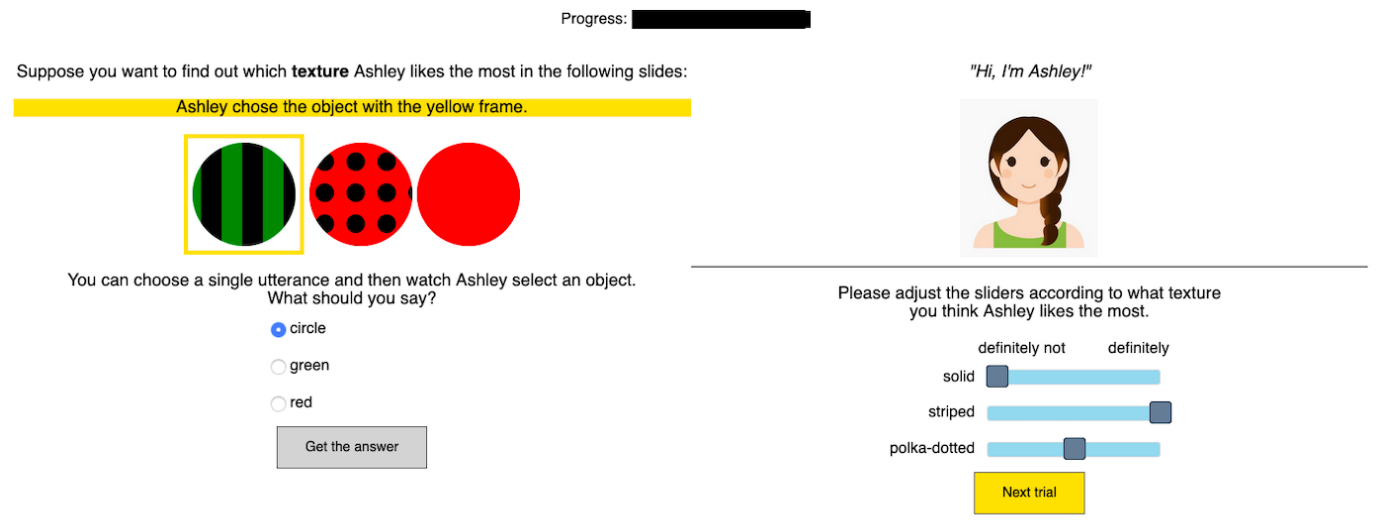

Figure 2: A sample trial. Each trial portrays a listener. The speaker (that is, the actual human participant) produces an utterance to refer to one of the objects. The simulated listener picks an object, which, as a result, gets marked by a yellow outline. The participant then evaluates which preferences of the listener may have led her to the particular object choice, specifying the inference by adjusting the sliders for each of the features. Please note that we fully focus on the preference inference process in this paper, leaving the analysis of the utterance choice behavior for future work.

On the basis of a post-test demographics questionnaire, we identified 98 participants as native speakers of English. 3 participants reported that they did not understand the instructions or were confused by the task. As a result, the data from 95 participants was included in the analysis. We obtained a confirmation from all the subjects that they agree to participate in the study.

\subsection{Design and methods}

We presented the subjects with a series of reference game scenarios modeled after Figure 1 from Frank and Goodman (2012). The experiment participant played the role of the speaker. Her task was to find out the preferences of a listener for a particular feature type, that is, for color, texture, or shape. We built in particular distributions of feature preferences giving a priority scale over the possible three feature values. The simulated listener picked the object that matched the highest value on the scale. For example, the listener my prefer red over green over blue when the color feature type is in focus, or she may prefer squares over rectangles over circles when the shape feature types is in focus. Objects were allowed to vary along three dimensions: color (blue, red, green), shape (cloud, circle, square), and texture (solid, striped, polka-dotted). The list of utterances contained the properties of the three objects present, excluding the utterances that correspond to the target feature type (e.g. texture in Figure 2). All trials contained at least one ambiguous utterance option.

Each trial consisted of 2 parts. First, the speaker had to select an utterance (left half of Figure 2) and watch the simulated listener pick an object. Listener's choices were driven by the literal semantics of the utterance, i.e. only red objects qualified as a possible choice following the utterance "red". For example, when the scale was "red $>$ green > blue", and all three colors were present in a scene and compatible with the utterance, the listener picked the red object. If only green and blue objects were present 
and qualified, the listener picked a green object.

In the second part of the trial (right half of Figure 2), the speaker adjusted the sliders guessing the listener's preferences. Then the experiment proceeded to the next trial with the same simulated listener and the same hierarchy of preferences. The sliders remained in their adjusted positions, so the participant could see which preference inferences she had made so far.

Participants completed a series of 4 blocks, each containing 4 trials. Listener names and which particular feature preferences the speaker needed to infer (color, shape or texture) were chosen randomly in each block but remained constant within a block.

\section{Results}

In the experiment, the task of the participants was to first choose an utterance, then to observe the simulated choice of an object by the listener, and ultimately to infer the listener's preferences by adjusting preference sliders (cf. Figure 2). Subjects performed these tasks in blocks of four trials. The behavioral data confirmed that participants were able to correctly infer the preferences of the simulated agent when choosing ambiguous over unambiguous utterances. When focusing the analysis on those participants that chose ambiguous utterances in at least 15 of the 16 trials (more than $1 / 3$ of all participants), in $72 \%$ of the blocks of four successive trials, the final feature type preference order matched perfectly with the true order. This percentage clearly improved over the four trials, yielding a percentage of $26 \%, 45 \%, 61 \%$, after having seen only the first, the first two, and the first three trials in a block, respectively. In the remaining $28 \%$ of the blocks of trials one feature ranking was swapped. In contrast, when analyzing the $1 / 3$ of the participants with the fewest choices of ambiguous utterances, no learning progress was observable over the four successive trials and the correct feature preference ranking was inferred in only $28 \%$ of the sets of trials.

We tested two main types of models with respect to the inferred preferences of the listener after observing her (simulated) object choice: an iterative Bayesian inference model that takes into account the posterior estimates from the previous trial in one block as the next prior, and a non-iterative model, which assumes uniform preference priors for all trials. Moreover, we compared the performance of these two models with a baseline model, which predicts uniform preference posteriors.

We optimized the two free parameters of the model-softness $\gamma$ and obedience $\beta$-at the global level, that is, we obtained a single pair of parameter estimates based on the data from all of the participants (two free model parameters). Given the parameters, we calculate posterior preference model predictions and compare them with the human data.

When comparing the model predictions for each trial with the human responses, simple linear regression statistics can be determined. In Figure 3, we plot the human data-slider values of individual trials-against the iterative model predictions. The iterative model captures a large proportion of variance in the human data $\left(r^{2}=0.558\right.$, $F(1,1138)=1437, p<0.001$ ), while the non-iterative model (Figure 4 ) is less accurate at predicting the data $\left(r^{2}=0.382, F(1,1138)=703.5, p<0.001\right)$.

To further assess the performance of the two models we can examine the KL values, measuring the divergence between the participants posterior preference estimates and the corresponding model's posterior estimates after optimization. Please note that we compare KL divergences and not log-likelihoods of the data given the model because the participants provide a preference distribution (by adjusting preference sliders, cf. 


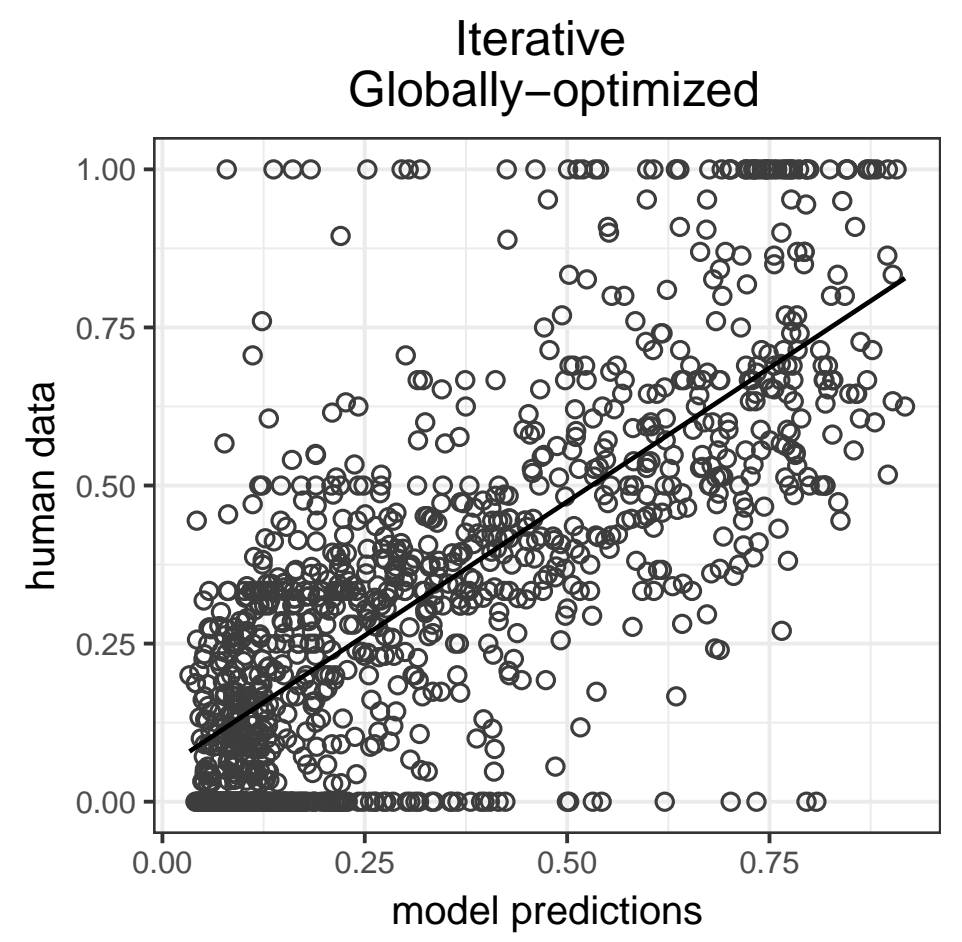

Figure 3: Iterative model predictions with softness $\gamma$ and obedience $\beta$ optimized globally. Each data point represents a single trial.

Figure 2) rather than indicating a concrete preference choice, as detailed above. As a result, the KL divergence values can be considered more informative than log likelihood values. Staying conservative, though, we treat the KL divergence values like log likelihood values in the following model comparisons.

As a lower baseline, we can use the KL divergence for the non-iterative uniform model, which yields a value of 558.7 when integrating all trials. $x$ Remember that a large KL divergence term indicates a poor fit of a model, seeing that the value corresponds to a difference measure between the participant data and the model's posterior estimates. The iterative model produces a KL value of 318.4, while the non-iterative one yields a value of 356.3. Clearly, both the iterative and non-iterative PSIRSA models strongly outperform the baseline. Since the KL divergence essentially corresponds to the negative log-likelihood between the model and the data, we can interpret the difference between two KL values as a Bayes factor. In our case we observe a Bayes factor of 37.9, which corresponds to very strong evidence in favor of the iterative model over the non-iterative ones on Jeffreys scale (Jeffreys, 1961; Lewandowsky \& Farrell, 2011).

When consideration the Bayesian Information Criterion (BIC) instead, the values need to be doubled and a penalty of $K \ln N$ needs to be added, where $N$ is the number of considered data points, which is equal to the number of participants times the number of trials, that is, $\ln N=\ln (95 * 16)=7.33$, and $K$ equals to the number of parameters, which is $K=2$ when comparing with the baseline model and $K=0$ when comparing the iterative with the non-iterative model. BIC differences above 20 are considered to 


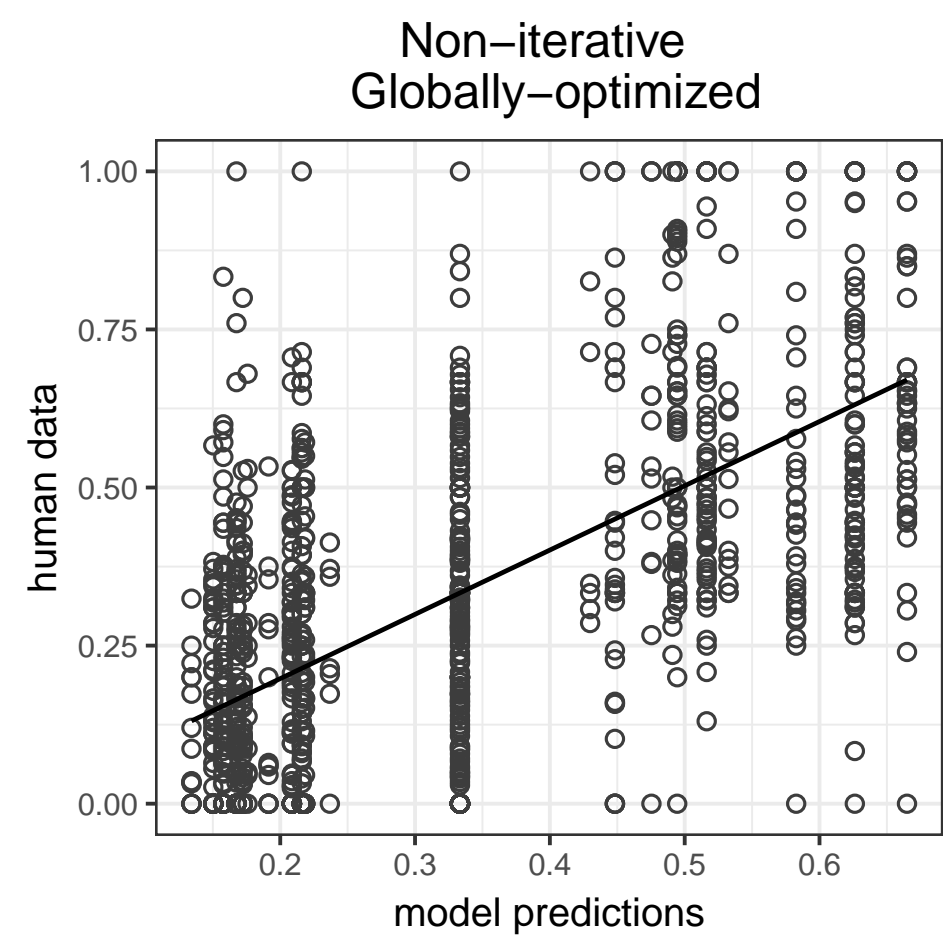

Figure 4: Non-Iterative model predictions with softness $\gamma$ and obedience $\beta$ optimized globally. Each datapoint represents a single trial.

offer strong evidence for the better model (cf. e.g. (Lewandowsky \& Farrell, 2011)). When comparing the non-iterative with the baseline model, we receive a difference of 397.47. The iterative model beats the baseline model by an even larger margin. The BIC value difference when comparing the iterative with the non-iterative model yields a difference that corresponds to twice the Bayes factor, that is, 75.8, confirming that the iterative model indeed models the human reasoning process more accurately than the non-iterative one.

We furthermore optimized the model's two parameters for each individual participant separately, which yields an even better KL value of 237.89. Although this difference may be considered not significant when compared to the globally optimized one due to the much larger number of free parameters ( 2 per participant, that is, $190 \mathrm{vs.}$ 2 in total for the global model optimization case above), it is still interesting to consider the resulting correlation plot. Figure 5 essentially confirms the fit of the globallyoptimized version, yielding an event better correlation $\left(r^{2}=0.673, F(1,1138)=2349\right.$, $p<0.001)$. Thus, while the individually optimized model appears to catch important individual differences, it adheres to the confirming trend that participants indeed pursue an iterative, approximately-Bayesian inference process. 


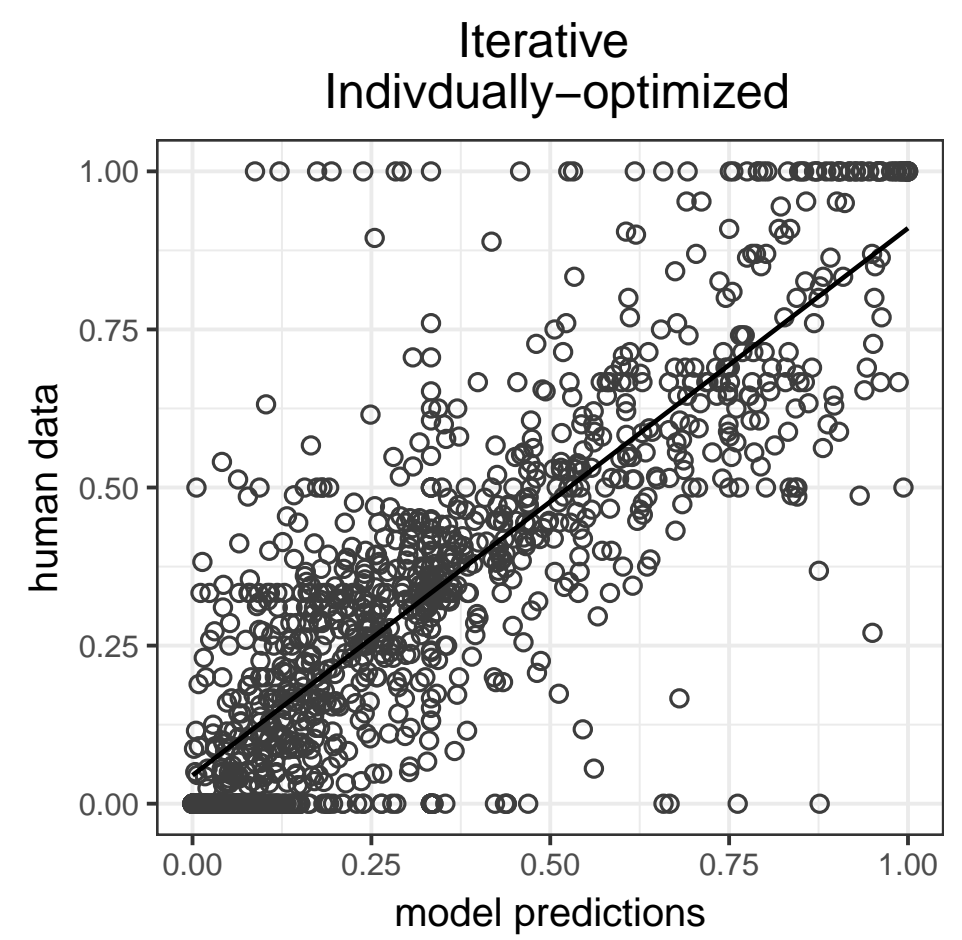

Figure 5: Iterative model predictions with softness $\gamma$ and obedience $\beta$ optimized individually for each participant. Each data point represents a single trial.

\section{Discussion}

In order to interpret an ambiguous utterance, the listener needs to draw on her prior knowledge to decide which interpretation is most likely. This prior knowledge, broadly viewed, can include personal preferences and beliefs, as well as the understanding of the current context. Listener choices are in that sense informative about the underlying priors of the listener.

We simulate the ambiguity resolution process with a Bayesian reasoning model that allows accumulation of evidence over a series of trials. The experimental data reveals that speakers are indeed able to infer hidden prior preferences by observing behavioral choices. Moreover, the model confirms that this process indeed unfolds in an cumulative manner, using the inferred posteriors as the next preference priors. Broadly speaking, this type of inference allows communicative partners to gain better understanding of each other, and to further anticipate how ambiguity may be resolved. The results are also in line with theories of social active inference (Friston \& Frith, 2015) but extend this rather general framework to the inference of motives and preferences, besides the interesting fact that dialogues may be played-out in a co-confirming, duet-like manner.

The presented computational model of the reasoning process takes into account iterative learning. It implements the idea that the accumulation of evidence may be realized by storing posteriors - here, of preferences, but in general of any decision biases, including knowledge, which the listener may have - and employing these stored posteriors as subsequent priors (here, over learned feature value preferences) to adjust 
them further in relevant, subsequent interactions.

While we do observe a better performance of the iterative model compared to the model that assumes uniform priors for each trial, we also acknowledge the fact that there is still substantial variance in the data that the model was unable to capture when globally or individually optimizing for two free parameters. A large part of this remaining difference may be explained by the fact that the model does not make any prior assumptions about the preference distribution of the listener. In contrast, the human participants can deduce after the first block of trials that the simulated listeners most likely have one most preferred feature value, one second most-preferred one, and one least preferred one. Thus, as long as the sliders are ordered accordingly, the answer can be considered correct from the participants' perspective, leaving lots of variance in the slider values. In fact, in order to model this ranked preference with PSIRSA, the preference estimates would need to be exponentially distributed on a rather extreme scale. In future work, we thus intend to enhance PSIRSA to either explicitly model the expectation of ranked preference distributions or to modify the preference encoding to a logarithmic scale, which may correspond more closely to the slider value interpretation of our human participants.

Nonetheless, the gathered evidence confirms that an iterative approximate Bayesian inference process is at play when interpreting choices of dialogue participants. Although the current scenario is certainly rather abstract, we are convinced that interpretation choices unfold in natural dialogues all the time, relying on assumptions about the conversation partner's intentions, knowledge, and preferences as well as the actual own ones. We thus hope to extend the proposed framework to even more natural settings in the near future, where true dialogues about a particular topic-such as a short story-unfold and conversation partners draw conclusions not only about untold parts of the story but also about the knowledge of their conversation partner when interpreting and generating respective sentences.

\section{Funding}

This project has been funded by the Deutsche Forschungsgemeinschaft (DFG, German Research Foundation)-Project number 198647426: Research Training Group 1808: Ambiguity - Production and Perception.

\section{References}

Chomsky, N. (2002). An interview on minimalism. In A. Belletti \& L. Rizzi (Eds.), On Nature and Language (p. 92-161). Cambridge: Cambridge University Press.

Clark, A. (2016). Surfing uncertainty: Prediction, action and the embodied mind. Oxford, UK: Oxford University Press.

Ferreira, V. S. (2008). Ambiguity, Accessibility, and a Division of Labor for Communicative Success. Psychology of Learning and Motivation: Advances in Research and Theory, 49, 209-246.

Frank, M. C., Goodman, N., Lai, P., \& Tenenbaum, J. (2009). Informative communication in word production and word learning. In Proceedings of the Annual Meeting of the Cognitive Science Society (Vol. 31).

Frank, M. C., \& Goodman, N. D. (2012). Predicting Pragmatic Reasoning in Language Games. Science, 336, 998-998. 
Franke, M. (2009). Signal to act: Game theory in pragmatics (Vol. DS-2009-11). Amsterdam: Institute for Logic, Language and Computation and Universiteit van Amsterdam.

Franke, M., \& Jäger, G. (2016). Probabilistic pragmatics, or why Bayes' rule is probably important for pragmatics. Zeitschrift für Sprachwissenschaft, 35(1), 3-44.

Friston, K., \& Frith, C. (2015). A Duet for one. Consciousness and Cognition, 36, 390-405.

Goodman, N. D., \& Frank, M. C. (2016). Pragmatic Language Interpretation as Probabilistic Inference. Trends in Cognitive Sciences, 20(11), 818-829.

Goodman, N. D., \& Stuhlmüller, A. (2013). Knowledge and implicature: Modeling language understanding as social cognition. Topics in cognitive science, 5(1), 173 184.

Grice, H. P. (1975). Logic and conversation. In P. Cole \& J. L. Morgan (Eds.), Syntax and Semantics 3: Speech Acts (p. 26-40). New York: Academic Press.

Hohwy, J. (2013). The predictive mind. Oxford, UK: Oxford University Press.

Jeffreys, H. (1961). Theory of probability, Clarendon. Oxford.

Jones, E. E., \& Davis, K. E. (1965). From acts to dispositions the attribution process in person perception. In Advances in experimental social psychology (Vol. 2, pp. 219-266). Elsevier.

Kelley, H. H. (1967). Attribution theory in social psychology. In Nebraska symposium on motivation.

Kelley, H. H., \& Stahelski, A. J. (1970). Social interaction basis of cooperators' and competitors' beliefs about others. Journal of personality and social psychology, 16(1), $66-91$.

Lewandowsky, S., \& Farrell, S. (2011). Computational modeling in cognition: Principles and practice. Thousand Oaks: Sage Publications.

Piantadosi, S. T., Tily, H., \& Gibson, E. (2012). The communicative function of ambiguity in language. Cognition, 122, 280-291.

Qing, C., \& Franke, M. (2015). Variations on a Bayesian Theme: Comparing Bayesian Models of Referential Reasoning. In H. Zeevat \& H.-C. Schmitz (Eds.), Bayesian Natural Language Semantics and Pragmatics (p. 201-220). Springer.

Sikos, L., Venhuizen, N., Drenhaus, H., \& Crocker, M. (2019, 04). Reevaluating Pragmatic Reasoning in Web-based Language Games. doi: 10.13140/RG.2.2.30535 .14249

Wasow, T. (2015). Ambiguity Avoidance is Overrated. In S. Winkler (Ed.), Ambiguity: Language and Communication (p. 29-47). de Gruyter. 